\title{
The Use of Fourier Analysis in the Interpretation of X-ray Line Broadening from Cold-Worked Iron and Molybdenum
}

\author{
By G. K. Williamson and R. E. Smallman* \\ Department of Physical and Theoretical Metallurgy, The University of Birmingham, England
}

(Received 16 January 1954)

\begin{abstract}
Measurements of the line shapes in the Debye-Scherrer spectra of iron and molybdenum, cold worked by filing, have been made using a Geiger-counter spectrometer. Fourier coefficients of the broadening functions have been calculated, using the coefficients for the annealed specimen as a measure of the instrumental broadening. The values of the r.m.s. strain deduced from the 110 and 220 reflexions do not coincide, but differ by $30-50 \%$. An investigation into the coefficients of theoretical line shapes has been made, and a similar effect has been found for the case of broadening due to a 'Cauchy' strain distribution. Equations which have previously been thought to make no a priori assumptions as to line shape are shown to hold only for very restricted cases not likely to occur in practice. The unavoidable numerical errors in calculating the coefficients from any profile resembling a 'Cauchy', even with better-than-average experimental accuracy, appear to make rigorous analysis impossible. A semi-empirical approach is suggested, which, at least for the iron and molybdenum results, allows an accuracy bracket to be placed on the interpretation. The reliability of some important previous results is discussed.
\end{abstract}

\section{Experimental procedure}

The samples were prepared first by hand filing 'Armco' iron wire and molybdenum sheet of commercial purity and then by sieving the filings through a 350 mesh per inch screen. Samples of both filings were annealed in vacuo, the iron at $650^{\circ} \mathrm{C}$. for $1 \mathrm{hr}$., and the molybdenum at $1375^{\circ} \mathrm{C}$. for $1 \mathrm{hr}$. Flat specimens were prepared by pressing the filings, moistened with dilute Canada balsam in xylol (about $5 \%$ ), into the specimen holder with a thin glass plate. Line shapes were recorded manually using a Geiger-counter spectrometer, measurements being taken every $2 \frac{1}{2}^{\prime}$ in $2 \theta$ and every $10^{\prime}$ or $15^{\prime}$ in $2 \theta$ on the background. All counts were for approximately $1 \mathrm{~min}$. as determined by a second monitoring counter. A correction for dead time losses has been applied to all results (Hall \& Williamson, 1952). Monochromatic radiation reflected from a bentcrystal monochromator has been used throughout; Co $K \alpha$ reflected from quartz $(10 \overline{1} 1)$ and generated at $50 \mathrm{kV}$.P. for the iron specimens, and $\mathrm{Cu} K \alpha$ reflected from aluminium (111), also generated at $50 \mathrm{kV}$.P. for the molybdenum.

The Fourier coefficients of the line shapes were calculated by the Stokes (1948) method from the graphically resolved low-angle half of the $\alpha_{1}$ component. It has been assumed that the annealed line shapes are an exact measure of the instrumental broadening and the coefficients of the broadening function are then the quotients of those from the coldworked and annealed specimen line shapes.

In order that the coefficients themselves could be

* Present address: A.E.R.E., Harwell, Didcot, Berks., England. interpreted using the methods of Warren \& Averbach $(1950,1952 a, b)$ without any change in scale, LipsonBeevers strips have not been used, but instead tables of $\cos 2 \pi n h$ were constructed at intervals in $h$ of 0.001 and for values of $n$ equal to $1,2,3,5,10,20,30,40$ and 60 . It is then possible to obtain directly values of $A_{n}$ if the abscissae of the intensity profiles are in terms of $h$, where, using Warren \& Averbach's notation,

$$
h+l_{0}=2 a_{3} \sin \theta / \lambda .
$$

For conformity, $a_{3}$ has been taken to equal $a$, the lattice parameter. The order of reflexion $l_{0}$ can be non integral and is related to the normal indices of reflexion, $H K L$, by

$$
l_{0}^{2}=H^{2}+K^{2}+L^{2} .
$$

Using this method, data can be obtained for adjacent cells ( $n=1$ and the lattice repeat distance, $a$, apart) instead of the more usual value of from 5-10 spacings (Warren \& Averbach, 1950, 1952a, $b$; Smith, 1953; McKeenhan \& Warren, 1953).

\section{Anomalies in the interpretation of the coefficients}

The coefficients have been interpreted assuming that the broadening is due to lattice strains only (preliminary line-broadening measurements indicated only slight particle-size broadening) where, using the equation given by Warren \& Averbach,

$$
A_{n}=\exp \left[-2 \pi^{2} l_{0}^{2} \overline{Z_{n}^{2}}\right] \text {. }
$$

Here $\overline{Z_{n}^{2}}$ is the mean square displacement of cells $n a_{3}$ apart along perpendiculars to the reflecting plane. 


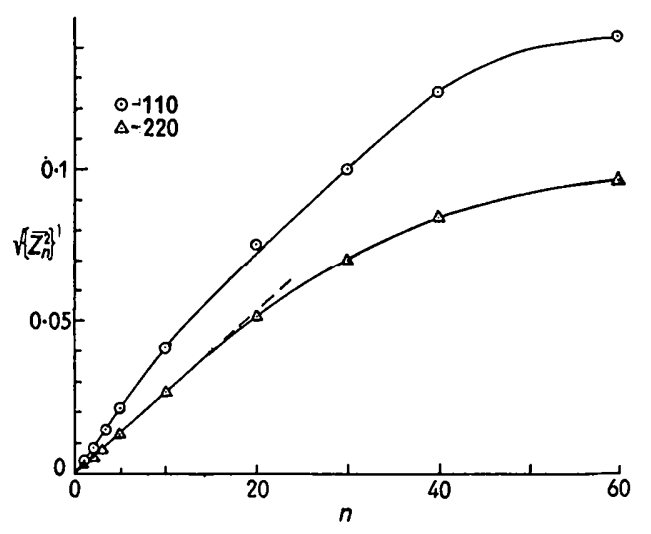

Fig. 1. The variation of $V\left\{\overline{Z_{n}^{2}}\right\}$ with $n$ (distance measured in units of the lattice parameter $a$ ) for cold-worked iron. There is a large discrepancy between the r.m.s. strains (represented by the initial slopes) deduced from the 110 and 220 reflexions.

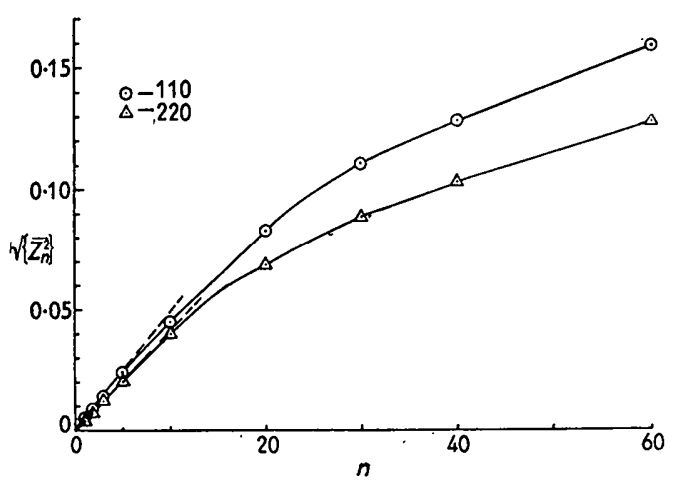

Fig. 2. The variation of $V\left\{\overline{Z_{n}^{2}}\right\}$ with $n$ for cold-worked molybdenum.

Figs. 1 and 2 show the variation of the r.m.s. displacement with $n$ for iron and molybdenum, as deduced from the 110 and 220 reflexions. In each case the data for the two reflexions should coincide, since they both represent the same physical effect; but instead two quite distinct curves are obtained, though they are of similar shape. Thus for iron the r.m.s. strains, given by the initial slopes of the plot Fig. 1, vary from 0.00265 (220 reflexion data) to 0.0041 (110 reflexion data), the ratio of the two values being 1.55 instead of the expected value of unity. For molybdenum (Fig. 2) the discrepancy is not quite so great, but it is still too serious to be dismissed without further investigation. Similar results have also been reported by both Warren \& Averbach $(1950,1952 a, b)$ and Smith (1953), and it has been suggested that equation (3) breaks down for large displacements. The results given in Figs. 1 and 2 are not consistent with this explanation since the discrepancy occurs even for the smallest displacements between adjacent cells $(n=1)$.

McKeenhan \& Warren (1953) have implicitly attributed the effect to small particle size and, in all cases so far reported, the anomaly is in the correct sense for this explanation to hold qualitatively. A similar effect would occur in line-broadening analysis (Williamson \& Hall, 1953) if the entire broadening produced by both particle size and strains were attributed to strains only. In fact, for Cauchy broadening functions, the differences in the apparent strains for the 110 and 220 reflexions would be $a_{3} / l_{0(220)} \varepsilon$, where $\varepsilon$ is the linear particle size. With the discrepancy reported above for molybdenum the particle size would have to be smaller than 200 spacings, a value which, though physically reasonable, is quite incompatible with the first Fourier coefficient $A_{1}$ for the 110 reflexion, namely $A_{1}=0.9992$ (for $\varepsilon=200$ spacings $A_{1}=0.995$ even in the absence of any strain effects). The results for molybdenum, however, have been analysed for particle-size broadening using Warren \& Averbach's (1952a) method in which the intercept at $l_{0}=0$ on the plot of $A_{n}\left(l_{0}\right)$ against $l_{0}^{2}$ gives the particle-size coefficients $A_{n}^{P}$, since

$$
\ln A_{n}=\ln A_{n}^{P}+\ln A_{n}^{D}=\ln A_{n}^{P}-2 \pi^{2} l_{0}^{2} \overline{Z_{n}^{2}} .
$$

The values $A_{n}^{P}$ obtained, using the coefficients $A_{n}$ for 110 and 220 reflexions, are shown plotted against $n$ in Fig. 3. A straight line fits the points for $n=10$,

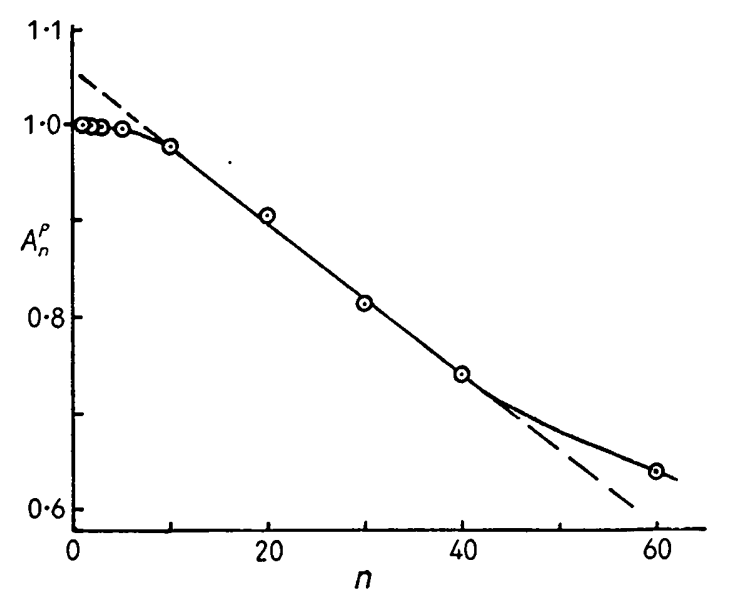

Fig. 3. A plot of the particle size coefficients $A_{n}^{P}$ agair ' $n$ for molybdenum. The intercept at $A_{n}^{P}=0$ indicates \& „rticle size of 150 spacings, or approximately $5 \times 10^{-6} \mathrm{c}$

20,30 and 40 , and indicates a particle size (the intercept on $n$ at $A_{n}^{P}=0$ ) of 150 spacings or $5 \times 10^{-6} \mathrm{~cm}$. The line neither intersects $A_{0}=1 \cdot 000$, as it must by definition, nor does it pass through the values for $n<10$, which would, if taken by themselves, indicate much larger particle sizes. Correction of the observed coefficients for this particle-size broadening leads to low-order strain coefficients greater than unity, which can have no physical meaning. Similar tendencies may be observed on the figures of Warren \& Averbach (1952a) and McKeenhan \& Warren (1953), though it is partially obscured by the paucity of data below $n=10(L \sim 25 \AA)$.

It was thought that many of these difficulties might be a result of numerical errors due to the omission of 
Table 1. Fourier coefficients from theoretical line shapes with a strain breadth $\xi=1 / 200 \mathrm{~V} \pi$

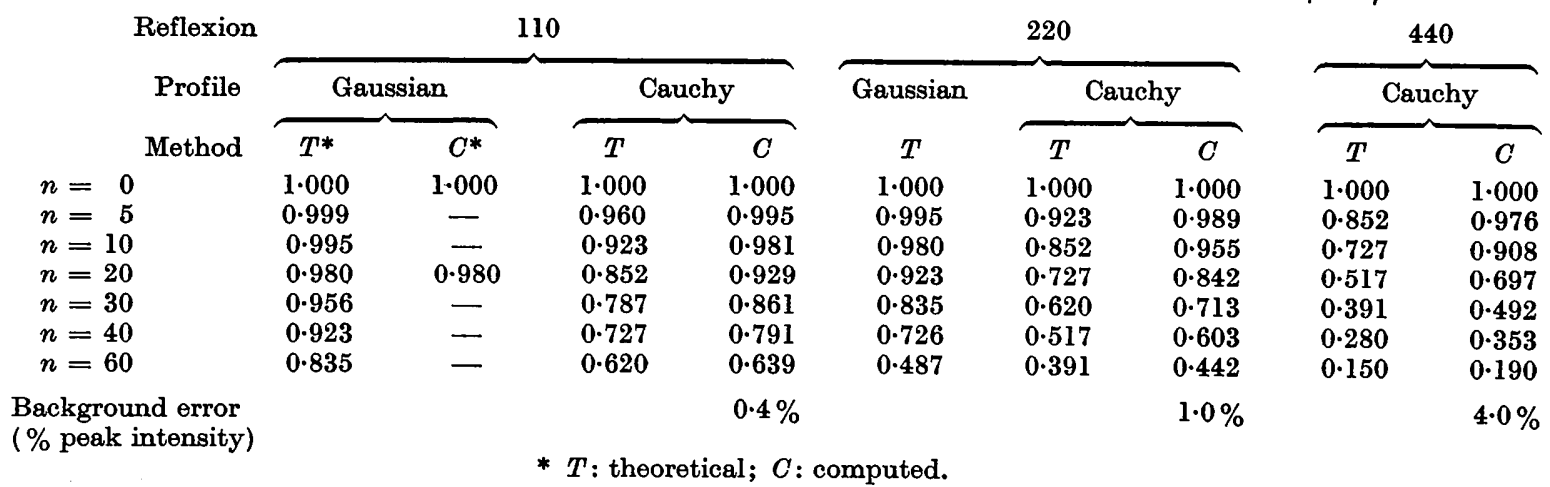

tails, as briefly discussed by Eastabrook \& Wilson (1952). The nature of these errors has been investigated for theoretical line shapes as described in the next section.

\section{Investigation of theoretical line profiles}

Gaussian line shapes were first chosen because equation (3) holds exactly. It has been assumed for simplicity that the broadening is due entirely to a strain distribution of the Gaussian-error-curve type, with an integral strain breadth $\xi=1 / 200 \sqrt{ } \pi$ (s.o that the mean square strain $\left.\overline{S^{2}}=\xi^{2} / 2 \pi\right)$, and that

$$
\overline{Z_{n}^{2}}=n^{2} \bar{S}^{2} \text {. }
$$

The line profiles have been calculated in terms of $h$, the reciprocal lattice variable, using

$$
I_{l_{0}}(h)=\exp \left[-\pi h^{2} / l_{0}^{2} \xi^{2}\right]
$$

for the $110\left(l_{0}=\sqrt{ } 2\right)$ and $220\left(l_{0}=2 \sqrt{ } 2\right)$ reflexions. Fourier coefficients have been obtained, as described in $\S 1$, by numerical summation. The computed coefficients agreed with those calculated from equation (3) within $0.1 \%$ and showed no anomalies in the plot of $\sqrt{ }\left\{\overline{Z_{n}^{2}}\right\}$ against $n$, for which data from both reflexions gave a linear plot, as expected from equation (5), and indicated the correct value of the mean square strain.

Some of the coefficients are listed in Table 1.

Cauchy profiles were next examined since these have very long tails, resembling those observed in practice, so that a more reasonable indication of the importance of tail errors is to be expected. Intensity profiles were calculated using the same value of $\xi$ as before for the 110,220 and 440 reflexions, using

$$
I_{l_{0}}(h)=\left\{1+\left(\pi h / l_{0} \xi\right)^{2}\right\}^{-1} \text {. }
$$

Line shapes have been cut off at too high a background level to simulate the effect of experimental errors. The magnitudes of the errors (Table 1) are probably much smaller than those made in practice, particularly for the 220 and 440 reflexions.

The computed coefficients (Table 1) will be seen to fall off much more rapidly with $n$ than do the coefficients for a Gaussian profile of identical integral breadth. Interpretation of these computed coefficients, using equation (3) for the r.m.s. displacement $V\left\{\overline{Z_{n}^{2}}\right\}$, is shown in Fig. 4, which is strikingly similar to the curves observed in practice (Figs. 1 and 2) particularly in the

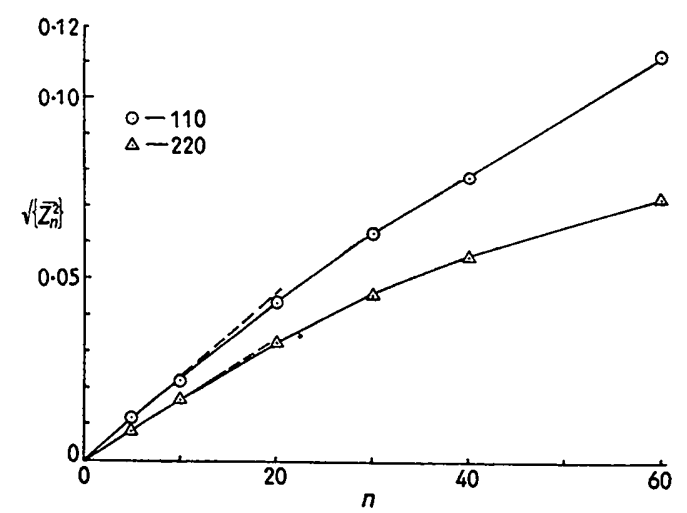

Fig. 4. A plot of $V\left\{\overline{Z_{n}^{2}}\right\}$ against $n$ deduced from the computed coefficients for theoretical line shapes with broadening produced by a Cauchy strain distribution. The resemblance to Figs. 1 and 2 is discussed in the text.

marked difference between the apparent mean square strains for the 110 and 220 reflexions. It is important to note that equation (5) predicts a linear relation between $V\left\{\overline{Z_{n}^{2}}\right\}$ and $n$, though this is not apparent in Fig. 4, which shows the usual, but in this case fortuitous, non linear relationship. The difference in slopes above $n=20$ is surprising because the tail errors are expected to be negligible above this point (Eastabrook \& Wilson, 1952).

The computed 'Cauchy strain' coefficients can be interpreted in terms of simultaneous particle-size and strain broadening even though the original profile represents strain broadening only. Thus the $\ln A_{n}$ versus $l_{0}^{2}$ plots (Fig. 5) give a fairly good straight line, though the fit of the points could be improved if the tail error for the 110 reflexion was reduced, or that for 220 increased. $A_{n}^{P}$ values, deduced by neglecting the results for the 440 reflexion, are shown plotted against 


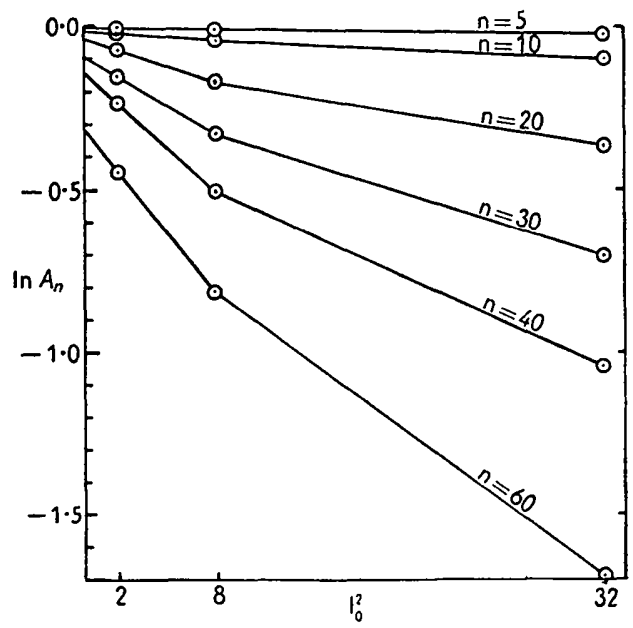

Fig. 5. A plot of $\ln A_{n}$ against $l_{0}^{2}$ for the computed coefficients from theoretical line shape broadened solely by a Cauchy strain distribution. The intercepts at $l_{0}^{2}=0$ lead to the particle-size plot shown in Fig. 6.

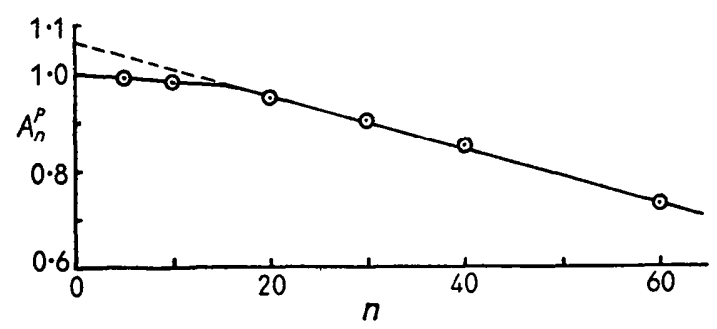

Fig. 6. A plot of the 'particle size' coefficients $A_{n}^{P}$ (deduced in Fig. 5) against $n$. A small particle size of 240 spacings is indicated even though the original profiles were calculated to correspond to an infinite particle size.

$n$ in Fig. 6, where a particle size of 240 spacings is indicated. As in practice, the plot does not intersect $A_{0}^{P}=1.000$ and the value of $A_{5}^{P}$, taken by itself, indicates a much larger particle size. Even smaller particle sizes could be obtained by neglecting the results for the 220 reflexion.

It is apparent, without further discussion, that the results for iron and molybdenum have an alternative explanation to that in terms of small particle size and strain if the strain distribution producing the broadening is a Cauchy profile, an explanation which appears to account for most of the anomalies which are otherwise obtained (Figs. 1, 2 and 3 ).

The correct values of the coefficients for the Cauchy strain profile can be found by direct integration. Solution of the integral equation for $A_{n}$ gives (Copson, 1935)

$$
A_{n}=\exp \left(-2 n l_{0} \xi\right) \text {. }
$$

This equation leads to the theoretical values for the coefficients listed in Table 1 . The only similarity between these coefficients and those for the Gaussian distribution is that they both obey the equality for 'strain' coefficients for the $l_{0}$ and $m l_{0}$ reflexions:

$$
A_{n}\left(l_{0}\right)=A_{n / m}\left(m l_{0}\right) \text {. }
$$

It should be noted that $A_{n}$ initially varies linearly with $n$, instead of with $n^{2}$ as for Gaussian profiles.

Equation (8) is adequate for most interpretations though it can usefully be written in terms of the r.m.s. strain if the necessary integration is cut off at some small strain instead of infinity. In the Fourier analysis the lines finish at strains of 0.5 , but for calculating stored energy the strain at which Hooke's law becomes invalid is the maximum reasonable value, and thus the limit is probably $0 \cdot 2$. In general, if the cut-off strain is $c$, then, if $c>3 \xi$,

$$
\left.\overline{\left(S^{2}\right.}\right)_{c}=2 c \xi / \pi^{2}
$$

is a good approximation, and thus for a cut-off strain of $0 \cdot 2$

Writing

$$
\left(\overline{S^{2}}\right)_{0.2}=2 \xi / 5 \pi^{2} .
$$

$$
\overline{Z_{n}^{2}}=n^{2} \bar{S}^{2}=2 n^{2} c \xi / \pi^{2},
$$

and substituting in equation (8), $A_{n}$ becomes

$$
A_{n}=\exp \left(-\pi^{2} l_{0} \overline{Z_{n}^{2}} / n c\right) \text {. }
$$

Equation (13) is important in so far as it emphasizes the limited application of equation (3), which was previously thought to be general. It can be seen that equation (3) does in fact make a priori assumptions as to the exact line shape and that the use of any analysis using it can only be justified if the line shape were shown to be a Gaussian error curve.

It is now possible to discuss more fully some of the consequences of interpreting coefficients from a Caurhy strain profile using equation (3). If $\left.V / \overline{Y_{n}^{2}}\right\}$ is the apparent r.m.s. displacement deduced on this basis, by equating (3) and (8) for $A_{n}$ we have

$$
\begin{gathered}
2 \pi^{2} l_{0}^{2}\left(\overline{Y_{n}^{2}}\right)=2 n l_{0} \xi, \\
V\left\{Y_{n}^{2}\right\}=\left(n \xi / l_{0} \pi^{2}\right)^{\frac{1}{2}} .
\end{gathered}
$$

Thus the plot of r.m.s. displacement against $n$ is a parabola (Fig. 7), instead of the true linear relation necessary by equation (11), and each order gives a

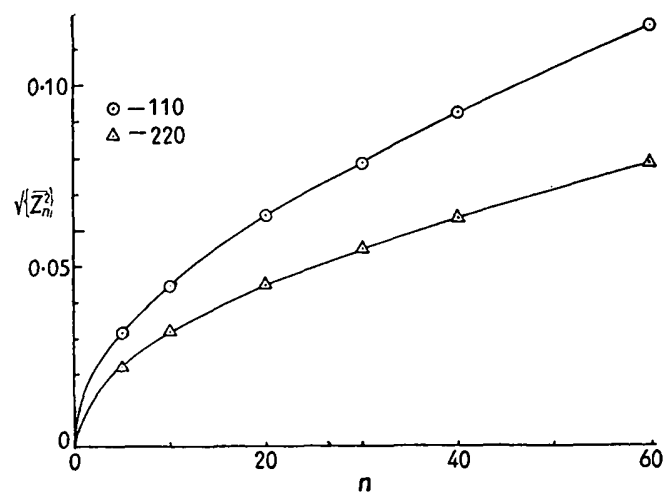

Fig. 7. A plot of $\sqrt{ }\left\{\overline{Z_{n}^{2}}\right\}$ against $n$ deduced from the theoretica coefficients for Cauchy profiles. 
different curve in which the scale of $n$ has changed in the ratios of the orders of reflexion; the apparent r.m.s. strain (at $n=0$ ) is infinity for all reflexions.

The effect of tail errors is to reduce the apparent r.m.s. strains to more reasonable values and to make the discrepancy between results for two orders less serious, as already shown in Fig. 4. Although these errors make the coefficients behave as if the line shape were much more nearly Gaussian, the coefficients do indicate to some extent the increased strain energy associated with the Cauchy profile. Thus both the theoretical and observed values of the mean square strain from the Gaussian profiles discussed above are $1.26 \times 10^{-6}$, that indicated by the computed coefficients interpreted as if the profile were Gaussian is 5.29 $\times 10^{-6}$ for the 110 , and $2.89 \times 10^{-6}$ for the 220 reflexions, while the true value for a Cauchy profile (cut-off strain $0 \cdot 2$ ) is $1.14 \times 10^{-4}$, an overall variation of 2 orders of magnitude.

The analysis for particle size (see equation (4)) also implicitly assumes that the strain distribution is a Gaussian. Theoretical values of the coefficients from a profile broadened only by a Cauchy strain distribution behave qualitatively on a plot of $\ln A_{n}$ against $l_{v}^{2}$ as if there were appreciable particle-size broadening. If only the first two orders of reflexion from one plane are considered the indicated particle size is in fact a simple function of the strain breadth:

$$
\varepsilon_{\text {apparent }}=a / \xi \text {. }
$$

In this case, however, the inclusion of results from a third reflexion invalidates the analysis since the $\ln A_{n}-l_{0}^{2}$ plot is parabolic instead of linear.

This test cannot yet be applied in practice since the presence of tail errors does tend to make the plot more nearly linear in addition to increasing the apparent particle-size broadening, as predicted by Eastabrook \& Wilson (see Figs. 5 and 6).

From the above it can be seen that the results presented in Figs. 1, 2 and 3 are more nearly consistent with the strain distribution being of the Cauchy type than with it being Gaussian, and hence alternative methods of interpretation are needed.

\section{Alternative methods of interpretation}

Many of the more obvious analytical approaches (such as a plot of $\ln \left(\ln A_{n}\right)$ against $\ln \left(l_{0}\right)$ cannot be applied because of the tail errors. These errors also make it unjustifiable to attach any physical significance to variations in the coefficients for $n<10$, though some interpretation of the higher coefficients is possible since they merely require renormalizing, i.e.

$$
\left(\ln A_{n}\right)_{\text {true }}=\left(\ln A_{n}\right)_{\text {obs. }}+\ln K,
$$

where $k=A_{0 \text { true }} / A_{0 \text { obs. }}$ Thus, since $A_{n}=A_{n}^{P} A_{n}^{D}$,

$$
\left(\ln \left(A_{n}\right)\right)_{\text {obs. }}=\ln A_{n}^{D}+\ln A_{n}^{P}-\ln K \text {. }
$$

A rigorous analysis to separate $A_{n}^{D}$ and $A_{n}^{P}$ is impossible unless the value of $K$ can be accurately assessed for each reflexion, apart from the difficulty of determining the appropriate function of $n$ and $l_{0}$ which defines $A_{n}^{D}$.

Possibly the best approach with the experimental accuracies now available is to attempt to bracket the required values and to assess the reliability of the possible interpretations. In the published work on particle-size broadening the line shapes are found to be intermediate between the two extremes of Cauchy and Gaussian (see for example Jones (1938) and Schoening, van Niekerk \& Haul, 1952).

For distortion broadening there is less evidence, but recent work (Averbach \& Warren, 1949; Hall \& Williamson, 1951) has shown that the broadening function has a broad base and very long tails, so that its behaviour will more nearly resemble that of a Cauchy than a Gaussian profile. Although it is not possible $a$ priori to rule out the possibility that some broadening functions lie outside this range (particularly in recovered materials) it seems to be a good working hypothesis to suggest that, in general, the true values would be bracketed by parallel analyses based on either equation (3) or (8). With results of moderate accuracy or better, equation (3) gives lower limiting values of both particle size and strain and equation (8) gives the upper limits. An estimate of the probability of each extreme can be obtained by comparing the behaviour of the observed coefficients on analysis, with those of the computed values for the appropriate theoretical profile. Figs. 4, 5 and 6 show the behaviour of the values for a Cauchy profile interpreted using equation (3), a behaviour surprisingly similar to that observed in practice.

The results of interpretations for 'strain' coefficients based on equation (8) are shown in Figs. 8 and 9. The use of equation (8) is preferable to the use of equation (13) since it does not involve the use of a cut-off strain $c$; the values of $n \xi$ can be regarded as

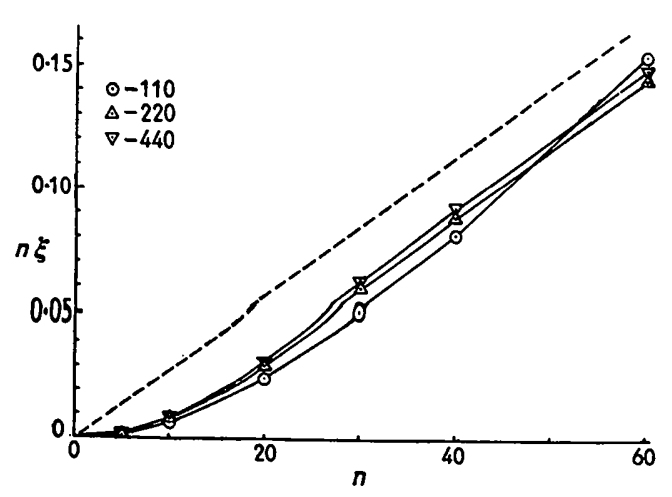

Fig. 8. The interpretation of the coefficients for Cauchy 'strain' profiles, using equation (8). The value of $n \xi$ represents a mean displacement and is plotted against $n$. The broken line is the theoretical variation, and results for the computed coefficients give a line of parallel slope above $n=15$. The initial deviation from this slope is due to numerical errors which are discussed in the text. 


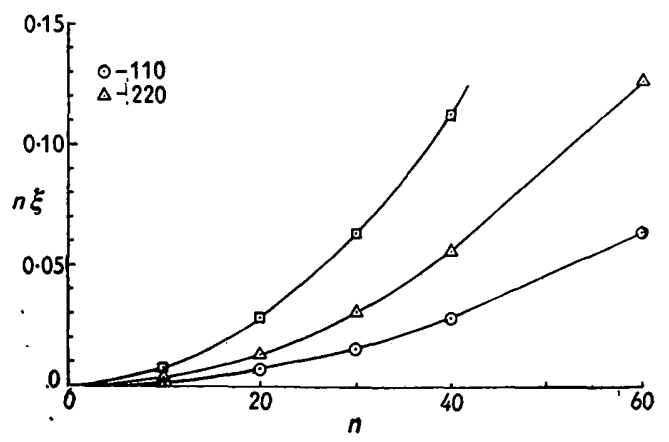

Fig. 9. A plot of $n \xi$ against $n$ for three 'reflexions' broadened by a Gaussian strain distribution.

a mean value of the displacement. For Cauchy profiles the plot of $n \xi$ versus $n$ is shown in Fig. 8. Values for all three reflexions indicate the same slope $(\xi)$ above $n=10$; this slope agrees closely with the theoretical value $\xi=1 / 200 \mathrm{~V} \pi$ assumed in calculating the original line shapes. The initial deviation from this slope is due entirely to tail errors and in practice no more physical significance can be justifiably attached to apparent increase in strain with $n$ in Fig. 8 than can be attached to the gradual decrease apparent in Figs. 1, 2, 4 and 7. The gradual decrease obtained in practice using equation (3) has commonly been given a physical interpretation, but this is completely unjustified until either the strain profile is shown to be a Gaussian, or until a very much more rigorous analysis is made. The interpretation of coefficients from a Gaussian profile using equation (8) (Fig. 9) leads once more to r.m.s. strain values which are a function of $l_{0}$, this anomaly being the reverse of the one which promoted the investigation (Figs. 1 and 2).

The plot of $n \xi$ against $n$ for the coefficients from the spectra of iron (using equation (8)) is shown in Fig. 10. The plot is linear above $n=10$, and comparisons of Fig. 10 with Figs. 8 and 9, and of Fig. 1

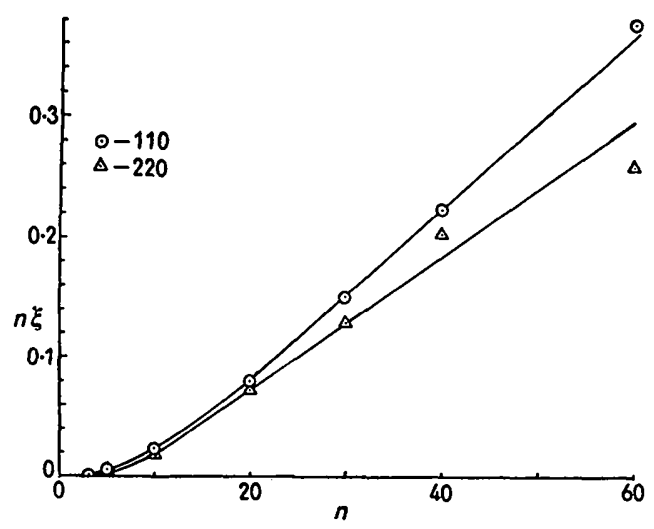

Fig. 10. A plot of $n \xi$ against $n$ for cold-worked iron.

with Fig. 4 suggest that in this case the broadening function is closer to a Cauchy than a Gaussian profile. The plots do not, however, coincide for different orders of reflexion, though the discrepancy is less than that in Fig. 1. This discrepancy might be due either to some particle-size broadening or to the strain-broadening function being even more extreme than a Cauchy. The particle-size broadening coefficients may be estimated on the assumption that the strain distribution is a Cauchy profile by plots of $\ln A_{n}$ against $l_{0}$ (instead of $l_{0}^{2}$ as in Fig. 5). Fig. 11 shows the plot of these coefficients against $n$ and indicates a particle size of $10^{-5} \mathrm{~cm}$. (350 spacings). The fall away from the best straight line below $n=15$ is quite reasonable since tail errors are expected for Cauchy profiles.

The interpretation of the molybdenum results, whilst giving a linear plot for $n>10$, gives a discrepancy in the indicated strain breadth which cannot be accounted for by any small-particle-size effect, and in the absence of other information this is consistent with the line shape being intermediate between the

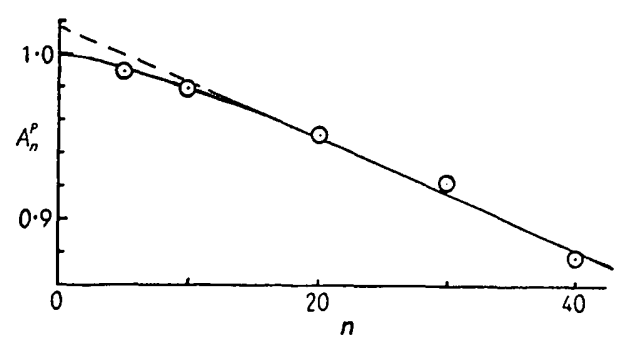

Fig. 11. A plot of $A_{n}^{P}$ against $n$ for cold-worked iron deduced by assuming a Cauchy strain distribution. The indicated particle size is $10^{-5} \mathrm{~cm}$.

two extremes. The upper limit to the particle size is thus infinity. It is of course not possible to attach any physical significance to the apparent rise of $\xi$ with $n$ in the range $n=0-15$ because for Cauchy line shapes tail errors are known to be serious and to cause just such an effect. On the other hand the broadening profile cannot be Gaussian, for with this profile numerical errors have a negligible effect and it is therefore not possible to account either for the difference between the theoretical curves (Fig. 9) and those for iron and molybdenum (Figs. 10 and 12), or for the anomalies at low $n$ values using equation (3) as discussed in $\S 2$. Thus the variation of r.m.s. strain

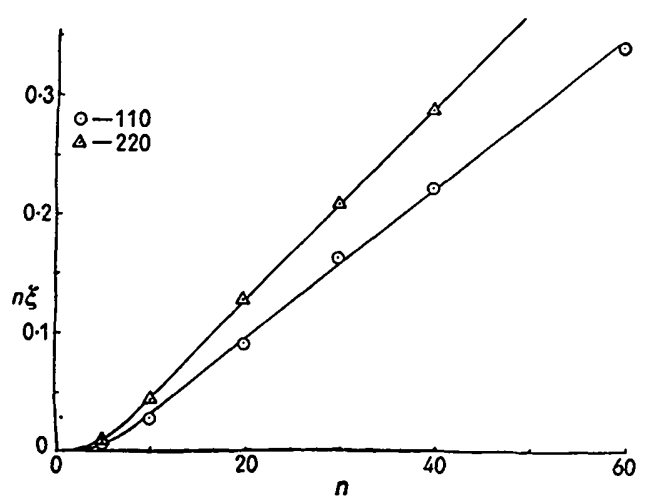

Fig. 12. A plot of $n \xi$ against $n$ for cold-worked molybdenum. 
Table 2. Limiting values of the mean square strain and particle sizes for iron and molybdenum

\begin{tabular}{|c|c|c|c|c|}
\hline & & & Part & cm.) \\
\hline & Cauchy & Gaussian & Cauchy & Gaussian \\
\hline $\begin{array}{l}\mathrm{Fe} \\
\text { Mo }\end{array}$ & $\begin{array}{c}0.8 \times 10^{-2} \\
0.8-0.94 \times 10^{-2}\end{array}$ & $\begin{array}{r}0.25-0.4 \times 10^{-2} \\
0.4-0.5 \times 10^{-2}\end{array}$ & $\begin{array}{c}1.0 \times 10^{-5} \\
\infty\end{array}$ & $\begin{array}{l}3.0 \times 10^{-6} \\
5 \cdot 0 \times 10^{-6}\end{array}$ \\
\hline
\end{tabular}

with $n$, as apparent in Figs. 1 and 2, since it is just the expected behaviour of coefficients from nonGaussian line shapes interpreted using equation (3), has no real physical significance; any such variation which may occur is certainly masked by the possible errors in the analysis, and Figs. 10 and 12 show that any such effect, if it occurs, is very small for cells greater than 10 spacings apart.

The limiting values of the mean square strain and particle sizes for the two specimens is shown in Table 2. The upper limits of the strain are to be preferred to the lower because Cauchy profiles are the more probable, but the limit for molybdenum is certainly slightly too high. Particle-size determination is, however, much less certain and, though both values for iron appear reasonable, the results for molybdenum have so large a range of values as to be inconclusive.

\section{Discussion}

The foregoing sections have indicated that it is not yet possible to use the method of analysing Fourier coefficients of the broadening function without some assumptions as to the exact form of the function itself. It should not be thought, however, that this makes the method any less powerful than those based on line broadening, for here too similar assumptions have to be made. The previous remarks in no way invalidate the 'Stokes' method for correcting for instrumental broadening, and, though the 'tail errors' discussed are returned to the tail, if the broadening function is calculated from the coefficients, the errors still persist in any line-broadening analysis. The Fourier analysis method does, of itself, partially compensate for the 'strain' profile if used for calculating stored energies, whereas line-broadening analysis makes no such correction. In this sense the Fourier analysis takes account of the line profile, and if sufficient data could be obtained exact limits could probably be set on the values of mean square strain.

Generally elastic anisotropy interferes seriously with the interpretation of results, but this is not so for tungsten, which is elastically isotropic. McKeenhan \& Warren have published a very full set of coefficients for thoriated tungsten from which they deduce a very small particle size. An analysis of their data on the Cauchy basis still indicates a small particle size though the particle size is somewhat larger than their value; the true value appears to lie in the range $200-600 \AA$. In this particular case particle-size broadening predominates and it is hence relatively insensitive to the assumed form of the strain distribution. This result is interesting because the more limited data of Williamson \& Hall (1953) for pure tungsten indicates a very much larger particle size.

Another two interpretations appear to be relatively unaffected by the previous discussion: they are (I) the analysis for fault broadening in deformed copper $2 \%$ silicon by Warren \& Averbach (1952a) and (2) the interpretation of the coefficients for a recovered metal by Williamson \& Hall (1953).

The most important interpretation which the present work has shown to be unjustified is the very attractive one that the strains decrease as the distance between cells increases. Though such an effect may occur, there is, as yet, no conclusive evidence for it since an alternative explanation in terms of experimental error always appears to be more plausible. Also the suggestion (Williamson \& Hall, 1953) that the 'cross over' of the Fourier coefficients from different orders indicated the presence of strains in the annealed specimen is no longer a valid argument, for much of the 'cross over', if not all, may be due to tail errors.

The mere existence of long tails on the strain distribution which give rise to the errors making exact analysis more difficult is, in itself, a very important result. It confirms the intensity work of Averbach \& Warren (1949), who reported long tails on the weak 400 reflexion, and the work of Hall \& Williamson (1951), who observed tails so long that they overlapped sufficiently to cause a detectable rise in the background level. These long tails lead naturally to the picture of the cold-worked state based on dislocations which has already been discussed elsewhere (William. son \& Hall, 1953).

We wish to acknowledge the encouragement of the late Prof. Hanson, Head of the Department of Metallurgy of the University of Birmingham, for financial support, a Wiggin Research Scholarship (R.E.S.) and an I.C.I. Research Fellowship (G.K.W.). We should also like to thank Dr M. A. Jaswon for valuable discussion.

\section{References}

Averbach, B. L. \& Warren, B. E. (1949). J. Appl. Phys. 20, 1066.

Copson, E. T. (1935). Theory of Functions of a Complex Variable, p. 130. Oxford: University Press.

Eastabrook, J. N. \& Wilson, A.J.C. (1952). Proc. Phys. Soc., Lond. B, 65, 67.

Hall, W. H. \& Williamson, G. K. (1951). Proc. Phys. Soc., Lond. B, 64, 946. 
HaLl, W. H. \& Williamson, G. K. (1952). J. Sci. Instrum. 29, 132.

Jones, F. W. (1938). Proc. Roy. Soc. A, 166, 16.

McKeenhan, M. \& Warren, B. E. (1953). J. Appl. Phys. 24, 52 .

Schoming, F. R. L., Niekerk, J. N. van \& Haut, R. A. W. (1952). Proc. Phys. Soc., Lond. B, 65, 528.

Sмiтн, R. A. (1953). J. Iron Steel Inst. 173, 147.

Stokes, A. R. (1948). Proc. Phys. Soc., Lond. 61, 382.
Warren, B. E. \& Averbach, B. L. (1950). J. Appl. Phys. 21, 595.

Warren, B. E. \& Averbach, B. L. (1952a). J. Appl. Phys. 23, 497.

Warren, B. E. \& Averbach, B. L. (1952b). Imperfections in Nearly Perfect Crystals, p. 152. New York: Wiley; London: Chapman and Hall.

Willtamson, G. K. \& Hall, W. H. (1953). Acta Metallurgica, 1, 22.

Acta Cryst. (1954). 7, 581

\title{
The Determination of Signs of Structure Factors from the Intensities
}

\author{
By W. CochraN \\ Crystallographic Laboratory, Cavendish Laboratory, Cambridge, England
}

(Received 21 May 1954)

\begin{abstract}
It is shown that in principle the magnitudes and signs of certain structure factors can be calculated from a sufficient range of $X$-ray intensities. The range provided by experiment will seldom be enough to make the results of practical value.
\end{abstract}

\section{Introduction}

A routine procedure which does not require previous knowledge of any signs has been developed by Hauptman \& Karle (1953) for determining the signs of the structure factors for space group $P \overline{1}$. Their claim that the procedure is valid for crystals of any complexity, given only a knowledge of a sufficient number of X-ray intensities, is, however, incorrect, as has been pointed out by Vand \& Pepinsky $(1953,1954)$ and by Cochran \& Woolfson (1954). In the following sections a method is described by which both the magnitudes and the signs of certain structure factors (one-eighth of the total for space group $P \overline{1}$ ) can in principle be calculated from a sufficient range of intensities. The results hold for centrosymmetric crystals and for some non-centrosymmetric crystals, but unfortunately the auxiliary conditions are so restrictive as to make the results of no value for the practical determination of any but the simplest crystal structures.

\section{Derivation for space group $\boldsymbol{P} \overline{\mathbf{1}}$}

The physical basis of the result is roughly as follows. Suppose that $N$ equal atoms per unit cell have coordinates $\pm \mathbf{r}_{i}, i=1,2, \ldots, \frac{1}{2} N$. The corresponding Patterson function has $N$ peaks of weight 1 at points $\pm 2 \mathbf{r}_{i}$ and $\frac{1}{2}\left(N^{2}-2 N\right)$ peaks of weight 2 at $\pm\left(\mathbf{r}_{i} \pm \mathbf{r}_{j}\right)$, $i \neq j$. In the squared Patterson function the weights of peaks belonging to these two classes are therefore 1 and 4 respectively. By multiplying the Patterson function by 2 , and subtracting from it a modification of the squared Patterson function, all peaks except those at $\pm 2 r_{i}$ can be cancelled out, and the positions of these remaining peaks bear an obvious relation to the atomic positions. These functions and operations have each a counterpart in reciprocal space, and when expressed in terms of structure factors the result is a relation between those structure factors whose indices are all even, and the intensities.

To simplify the derivation, we take the atoms to have scattering factors $f=1$ for all values of $s=$ $2 \sin \theta / \lambda$, that is, they are point atoms. The peaks of the Patterson function then have scattering factors (more correctly, Fourier transforms) $f^{2}=1$, from which it follows that the density distribution in an atomic peak and in a Patterson peak is the same. We now define

$$
\begin{gathered}
F(\mathbf{h})=2 \sum_{j=1}^{\frac{1}{2} N} \cos 2 \pi \mathbf{h} \cdot \mathbf{r}_{j}, \\
\varrho(\mathbf{r})=\sum_{s<s_{0}} F(\mathbf{h}) \cos 2 \pi \mathbf{h} \cdot \mathbf{r}, \\
P(\mathbf{r})=\sum_{s<s_{0}} F^{2}(\mathbf{h}) \cos 2 \pi \mathbf{h} \cdot \mathbf{r} .
\end{gathered}
$$

From (1) it may be shown that

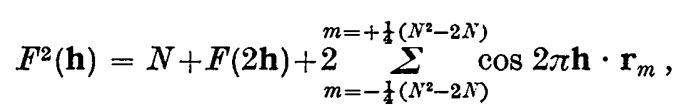

where $\mathbf{r}_{m}=\mathbf{r}_{i}-\mathbf{r}_{j}, i \neq j$.

The summation over $\mathbf{h}$ is over all reciprocal-lattice points which lie inside a sphere of radius $s_{0}$. (The value of $s_{0}$ will be considered later.)

We now define

$$
\varrho^{2}(\mathbf{r})=\sum_{s<2 s_{0}} G(\mathbf{h}) \cos 2 \pi \mathbf{h} \cdot \mathbf{r} .
$$

It then follows (Sayre, 1952) that 